\title{
The Effect of Prolonged Standing on Touch Sensitivity Threshold of the Foot: A Pilot Study
}

\author{
Neal E. Wiggermann, PhD, Robert A. Werner, MD, MS, W. Monroe Keyserling, PhD
}

Objective: To determine the effect of prolonged standing on touch sensitivity of the foot. Design: An observational study with replications.

Setting: University laboratory.

Participants: Ten healthy college students ( 5 men and 5 women), with a mean \pm SD age of $23.5 \pm 4.1$ years and body mass of $67.4 \pm 12.6 \mathrm{~kg}$.

Methods: Semmes-Weinstein monofilament tests were administered to 12 locations on the dorsal and plantar surfaces of the foot before and after 4 hours of standing. These locations were formed into several groupings (toes, metatarsal heads, midfoot, heel, all plantar sites, all dorsal sites), and paired $t$-tests were used to test for significant changes in sensitivity threshold after standing.

Main Outcome Measurement: The difference between sensitivity thresholds measured before and after standing for different locations on the foot.

Results: The average of all sensitivity thresholds on the plantar surface of the foot decreased (indicating increased sensitivity) from 0.56 to $0.36 \mathrm{~g}(P<.01)$ after 4 hours of prolonged standing. This change in threshold equated to a difference of 1 SemmesWeinstein monofilament level. Changes in the sensitivity threshold of the dorsal aspect of the foot were not significant.

Conclusions: Analysis of the results suggests that the plantar foot has greater sensitivity to touch after prolonged standing. These findings may be useful for identifying potential unintended bias in clinical touch sensitivity testing. Future research is necessary to understand the underlying mechanisms for this sensitivity change and to determine the onset and recovery times for sensitivity changes.

PM R 2012;4:117-122

\section{INTRODUCTION}

Peripheral neuropathy is a disorder of the peripheral sensory, motor, and/or autonomic nerves. There is a high prevalence of peripheral neuropathy in persons who have longstanding diabetes, with more than 50\% developing the disorder [1]. The most common form of peripheral neuropathy is distal symmetric polyneuropathy, which affects the longest nerves first and progresses proximally [1]. This polyneuropathy affects the A- $\beta$ nerves, which detect touch sensation, and is characterized by tingling, numbness, and pain [2]. Prompt diagnosis is important for managing the disease and preventing complications that result in foot ulcers [3] and amputation [4].

Assessment of touch sensation through psychosomatosensory threshold tests provides a rapid, comfortable, and inexpensive assessment of sensory function. This is clinically important in the evaluation of patients at risk for peripheral neuropathy. The most commonly used psychosomatosensory threshold test is the Semmes-Weinstein Monofilament (SWM) test, which has demonstrated effectiveness in detecting the risk for foot ulceration $[5,6]$. The nylon Semmes-Weinstein monofilaments vary in stiffness to allow determination of the threshold for touch sensitivity. SWM testing is commonly used in 2 ways, either by using a single filament to test for sensitivity at a critical level or by testing with a range of filaments to determine an exact sensitivity threshold. Much clinical research into the use of SWM testing has focused on using a single monofilament, the 5.07 level, which corresponds to $10 \mathrm{~g}$, for this prediction of risk for foot ulceration.

N.E.W. Center for Ergonomics, Industrial and Operations Engineering, University of Michigan, Ann Arbor, Ml. Address correspondence to: N.E.W.; e-mail: newiggermann@gmail.com Disclosure: 8, Training Grant T42 OH 008455 (Centers for Disease Control and Prevention/ National Institute for Occupational Safety and Health)

R.A.W. Department of Physical Medicine and Rehabilitation, University of Michigan Health System, Ann Arbor, MI

Disclosure: nothing to disclose

W.M.K. Center for Ergonomics, Industrial and Operations Engineering, University of Michigan, Ann Arbor, Ml

Disclosure: nothing to disclose

Disclosure Key can be found on the Table of Contents and at www.pmrjournal.org

Submitted for publication January 6, 2011; accepted November 8, 2011 
Table 1. Specified and measured values of Semmes-Weinstein monofilaments

\begin{tabular}{cccrc}
\hline Filament & Diameter $(\mathbf{m m})$ & $\begin{array}{c}\text { Specified Force, } \\
\text { Semmes-Weinstein (g) }\end{array}$ & $\begin{array}{c}\text { Specified Force, } \\
\text { Manufacturer (g) }\end{array}$ & $\begin{array}{c}\text { Measured } \\
\text { Force (g) }\end{array}$ \\
\hline 2.83 & 0.14 & 0.07 & 0.05 & $0.06(0.01)$ \\
3.22 & 0.16 & 0.16 & 0.20 & $0.15(0.01)$ \\
3.61 & 0.18 & 0.40 & - & $0.24(0.01)$ \\
3.84 & 0.21 & 0.60 & - & $0.42(0.01)$ \\
4.08 & 0.24 & 1.00 & - & $0.67(0.01)$ \\
4.17 & 0.27 & 1.40 & 2.00 & $1.09(0.03)$ \\
4.31 & 0.33 & 2.00 & $2.43(0.06)$ \\
\hline
\end{tabular}

Measured force values show mean (standard deviation).

Substantial research has been performed to determine the reliability and validity of measures of touch sensitivity [6-8], but, to our knowledge, there is no research that has investigated temporal variability of foot sensitivity itself, including the effects of exposure to activities of daily living. The objective of our research was to determine the effect of prolonged standing on touch sensitivity of the foot.

\section{MATERIALS AND METHODS}

\section{Measurement Instrument}

Semmes-Weinstein monofilaments were selected as the measurement instrument (supplied by Timely Neuropathy Testing LLC, Ventress, LA). The monofilaments were calibrated by N.E.W. by using an electronic gram scale (V-200; Acculab, Bohemia, NY). Calibration has been recommended due to variation in forces produced by each monofilament as a result of the manufacturing process and the temperature and humidity when used [9]. The forces produced by the monofilaments used in this experiment are presented in Table 1. Measured values were recorded as the average of 3 measurements after 2 cycles of bending to "break-in" each monofilament [10].

\section{Experimental Design}

Our research was part of a larger experiment that included 10 subjects ( 5 men, 5 women) with a mean \pm SD age of $23.5 \pm$ 4.1 years and a mean (SD) body mass of $67.4 \pm 12.6 \mathrm{~kg}$. The subjects reported no history of peripheral neuropathy, numbness or tingling in the feet, or diabetes. Approval for use of human subjects for this experiment was obtained from the university's institutional review board. The subjects stood in a climate-controlled room for 4 hours, interrupted by a 10-minute seated break after the first 2 hours. All the subjects wore the same brand and model of athletic shoes and socks. The purpose of this larger experiment was to measure the effect of antifatigue mats on discomfort during prolonged standing. SWM foot sensitivity measures were made immediately before and after each of 42 experimental sessions. Each subject participated in 3-6 sessions in which the proto- col remained unchanged except for the flooring surface used (a hard linoleum tile floor and antifatigue mats).

\section{Testing Procedure}

SWM sensory testing was conducted in a manner similar to that described by Hodge et al [11]. When the SWM test was administered, the participants removed their shoes and socks, closed their eyes, and returned an affirmative response when a filament was sensed. Sensitivity was tested at 8 sites on the plantar surface and at 4 sites on the dorsal surface of the dominant foot (shown in Figure 1). A variety of locations around the foot were chosen from sites identified in previous publications to be significant for providing protective sensation for the development of diabetic ulcers $[7,12]$. However, due to the large number of measurements performed, the
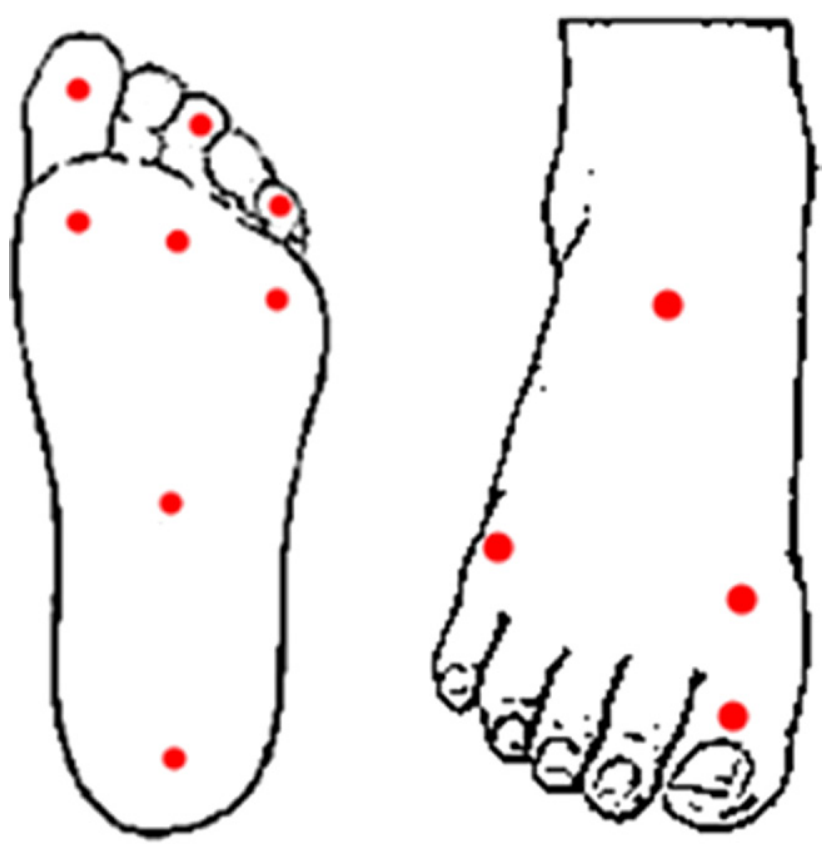

Figure 1. Locations tested on plantar (left) and dorsal (right) surface of the foot. 
number of locations was limited to reduce the overall testing time.

Locations were tested in random order, with irregular pauses between applications. Whenever possible, calluses and hair at the testing locations were avoided. At each site, the monofilament was gently pressed perpendicularly against the skin surface until the filament bowed. Pressure was held for approximately 1.5 seconds and then removed. If the subject did not elicit a positive sensation response during the initial test with a filament at a given site, then a second application was given, following the random order. A positive response was recorded if sensation was reported for at least one of the two applications with a given filament. Because of the irregular pauses between applications, no null stimulus condition was used. If, after an application, a response was unusually early or late, then participants were asked to identify the location of the sensation to rule out false-positive responses. Testing began with 2.83 as the thinnest filament level, and filaments were applied in ascending sequence until a single level was felt at all 12 sites on the foot.

With this protocol, the sensitivity threshold for all 12 locations could be tested in approximately 5 minutes. Speed was important because the objective of the study was to measure temporal changes in touch sensitivity, and the rate of recovery to normative values after prolonged standing is unknown. In addition, the SWM requires active concentration by the subject, and, after 4 hours of standing, boredom and fatigue associated with a long test was a concern. For these reasons, more sophisticated sensitivity threshold testing methods, such as stepping algorithms, which can average up to 5 minutes per testing location $[13,14]$, were not used.

\section{Data Analysis}

For the purpose of numerical analysis, SWM levels were converted to units of $\mathrm{g}$. This simplified statistical analysis allowed the use of arithmetic operations (SWMs are rated on a logarithmic scale) and enabled the use of the measured force values of the filaments determined during calibration
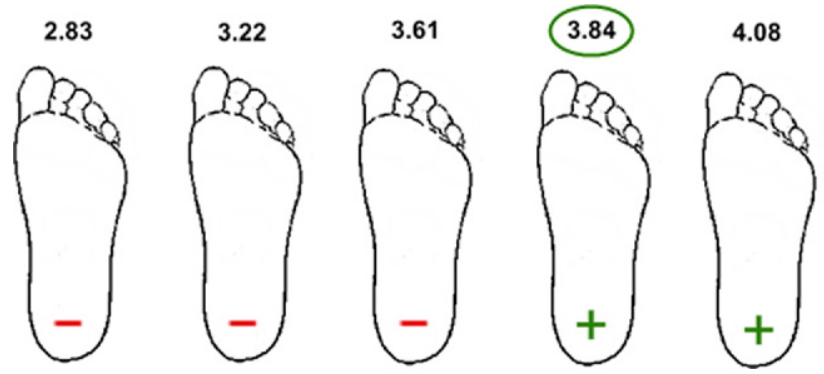

Figure 2. Example of a common observation for the heel. The site was not felt at the first 3 levels, and was first felt at 3.84. The heel location is therefore scored at .42 grams, the calibrated force corresponding to the 3.84 monofilament.

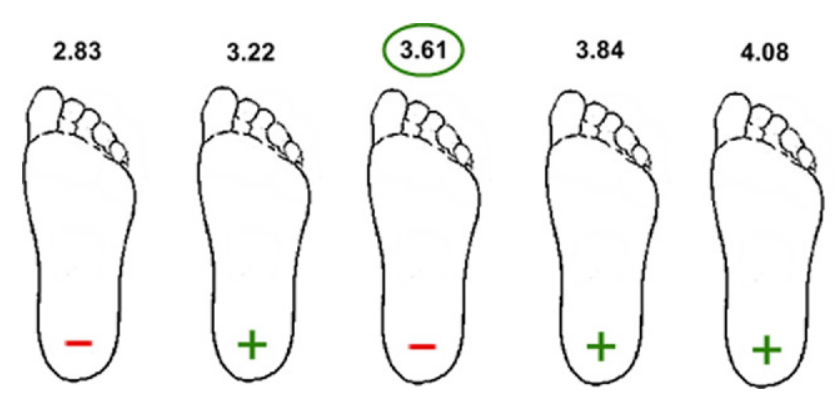

Figure 3. Example of an observation for the heel in which a filament is sensed at a lower level, and missed at the next higher level. Scoring is adjusted by increasing the lowest sensitivity felt (3.22) by the number of levels missed (in this case, 1) thus the force corresponding to filament level 3.61 is scored.

(shown in Table 1). To assign a numerical value to the sensitivity of a location, the force that corresponded to the thinnest monofilament that generated a positive response generally was scored. Usually, when a monofilament was sensed at a site, all higher levels of the monofilament also were sensed at that site (Figure 2).

In some cases ( $16 \%$ of total measurements), a monofilament was sensed at a lower level but was not felt at a higher level. In these cases, it was decided that recording the lowest sensed filament level while ignoring the fact that a subsequent filament was not sensed could result in an underestimation of the sensitivity threshold. However, recording the first filament sensed after the "gap" could overestimate the sensitivity threshold. To address this problem, when a gap occurred between sensed filament levels, the sensitivity threshold recorded was increased from the lowest level felt. If the gap consisted of one level, then the recorded threshold was increased by one filament level, and, if the gap contained 2 filament levels, then the threshold was increased by 2 levels. This scoring system is demonstrated in Figure 3.

After values for individual testing sites were determined, data from physiologically similar locations were combined into 5 regions to more easily compare different areas of the foot. The hallux, third toe, and fifth toe testing sites were averaged (toes), as were the sites under the first, third, and fifth metatarsal heads (metatarsals). Data from the 4 dorsal sites were averaged (dorsal), whereas the "heel" and "midfoot" sites were analyzed as single locations. In addition, a "plantar average" was calculated by taking the mean of the 4 plantar regions (toes, metatarsals, midfoot, heel). Paired $t$ tests were performed to compare the sensitivity values before and after prolonged standing for each region and for the plantar average.

\section{RESULTS}

The paired $t$-test of plantar sensitivity showed that 4 hours of standing significantly lowered the force threshold for sensing 




Figure 4. Mean Sensitivity thresholds ( $g$ ) before and after 4 hours of standing for regions of the foot. Error bars represent standard error of the mean. $P$-values are for paired t-tests comparing before and after standing.

a monofilament (increased sensitivity) for all plantar sites $(P<.05)$. However, there was no evidence of a significant change in the sensitivity threshold of the dorsal surface of the foot $(P=.12)$. Means and standard errors for sensitivity thresholds before and after 4 hours of standing are shown in Figure 4 . The mean decrease in average plantar sensitivity threshold was $0.20 \mathrm{~g}(P<.01)$, from $0.56 \mathrm{~g}$ before standing to $0.36 \mathrm{~g}$ after standing. The heel and metatarsals showed the largest decrease in threshold (largest increase in sensitivity), with differences of $0.37 \mathrm{~g}(P=.01)$ and $0.22 \mathrm{~g}(P=.01)$, respectively, whereas the midfoot demonstrated the lowest decrease in threshold (smallest increase in sensitivity), $0.04 \mathrm{~g}$ $(P=.03)$. For the toes, metatarsals, and dorsal regions in which sites were averaged, each individual site followed the same trend as the region for which it was included.

\section{DISCUSSION}

To our knowledge, the increased plantar sensitivity (ie, the decreased thresholds) after prolonged standing is previously undocumented. This finding may have implications for interpreting results of diabetic neuropathy testing $[6,8]$. More research is necessary to accurately quantify this phenomenon with respect to the duration of standing time, to investigate whether diabetic populations are affected, and to understand the underlying cause of the sensitivity changes.

\section{Testing Procedure}

This study introduces a modified touch sensitivity testing protocol that allows for sensitivity threshold to be measured for 12 sites on the foot in about 5 minutes. Because the objective of this study was to evaluate touch sensitivity threshold changes with respect to time, this rapid testing protocol was necessary to limit the time during which sensitivity could change while the test was being administered. This method reduced anticipation bias by testing foot locations in a random order and reduced risk of false-positive stimulus detection by using irregular pauses between the applications of each monofilament [11]. Although it is possible that this testing method is less accurate or more variable than more time-consuming threshold-detection methods $[13,14]$, the results demonstrate that it is an effective protocol for measuring relative changes in touch sensitivity. A validation of this rapid testing method may support its widespread use in future research applications that involve temporal changes in touch sensitivity or in clinical settings in which time for assessment may be limited.

\section{Limitations}

This study measured changes in touch sensitivity of the foot over several replications while controlling for footwear and activity during prolonged standing. However, this research was a pilot study as part of a larger experiment and sought 
merely to establish whether prolonged standing would affect changes in plantar touch sensitivity. The experiment included only young healthy subjects, so future experimentation will be necessary to determine whether these findings extend to older populations and to patients with diabetes at risk for peripheral neuropathy. This experiment also exposed subjects exclusively to standing. It is unknown whether similar results would be observed for prolonged periods of walking or for mixed standing and walking.

Although the subjects were encouraged not to stand or walk for long periods before the experiment, their activity before each experimental session was not standardized, nor was footwear worn before the experiment standardized. Testing sites were not marked by the investigators, so it is possible that there was some variation in testing location for each site.

This experiment on its own is insufficient to explain the mechanism for change in sensitivity, but analysis of these results suggests that the primary cause may be tissue compression, possibly resulting in hyperalgesia. Although all plantar surfaces demonstrated significant change in sensitivity, the dorsal surface, presumably subject to very little compression, did not show significant change.

Venous pooling in the legs is a condition that may cause a change in sensitivity [15]; however, if this were the case in this study, then a significant change would have been expected for the dorsal surface in addition to the plantar locations. The effects of skin temperature and moisture could not be ruled out by this study. Skin temperature has been shown to affect sensitivity [16], and skin moisture may affect sensitivity by altering the mechanical properties of the skin $[17,18]$. This experiment was conducted in a climate-controlled laboratory, but skin temperature and moisture were not measured at the time of testing. These effects are likely small because the dorsal aspect of the foot did not demonstrate significant sensitivity changes with prolonged standing and would presumably experience similar moisture and temperature. However, moisture and temperature differences may have been possible due to the mesh fabric top of the shoes, which may have allowed evaporation.

\section{Future Work}

To identify the causes of changes in plantar sensitivity, future studies should measure the skin temperature and moisture of the feet when measuring touch sensitivity. If sustained tissue compression is indeed the primary cause of change in sensitivity, then measures of changes in skin stiffness and fat pad thicknesses also may help to identify the underlying mechanics for this sensitivity change. Future research also should control for activity and footwear used before the experiment. More accurate calibration of monofilaments can also be performed by using strain gauge transducers [9]. Finally, touch sensitivity should be measured more frequently during and after standing to determine the onset and recovery times for sensitivity changes, which will provide physicians with a potential corrective factor for SWM testing of patients who have been standing.

\section{Implications for Clinical Practice}

The greatest change in sensitivity threshold occurred at the heel, where the mean sensitivity threshold dropped from 0.95 to $0.58 \mathrm{~g}$ after standing. A $0.95 \mathrm{~g}$ corresponds very nearly to the 4.08 filament level, whereas $0.58 \mathrm{~g}$ is nearly equal to the 3.84 filament level (Table 1). Therefore, this change equates to reduction in threshold of approximately one filament level. By using the approach just described, sensitivity threshold changes were computed in terms of filament levels for all locations. However, interpolation was used to make exact conversions because beginning and ending threshold forces were never exactly equal to specified filament levels. Thresholds were reduced an equivalent of 0.9 filament levels for the toes, 0.8 for the metatarsals, 0.4 for the midfoot, 1.0 for the heel, and 1.0 levels for the plantar average.

An inability to sense an SWM level of $5.07(10 \mathrm{~g})$ is a clinically accepted indicator of risk for foot ulceration in patients with diabetes [3]. The results of this experiment suggest that touch sensitivity testing may result in a falsenegative finding if the patient is tested after having spent a prolonged time on his or her feet. This standing may result in a reduction of sensitivity threshold, and the patient may be thought to have normal sensation when, in fact, sensation is impaired. If these same results are observed in patients with diabetes, then this might require testing patients who have been standing for extended periods by using a different filament, such as the 4.93 (8 g), which is one level below the 5.07 filament. Further research into the temporal variability of touch sensitivity with respect to postural activities will be useful for developing modifications to testing procedures to address potential unintended bias in clinical touch sensitivity testing.

\section{REFERENCES}

1. Eastman RC. Neuropathy in diabetes. In: National Diabetes Data Group, ed. Diabetes in America. 2nd ed. Bethesda, MD: National Institute of Diabetes and Digestive Kidney Diseases; 1995, 339-348.

2. Woolf CJ, Doubell TP. The pathophysiology of chronic pain: Increased sensitivity to low threshold Ab-fibre inputs. Curr Opin Neurobiol $1994 ; 4: 525-534$

3. McNeely MJ, Boyko EJ, Ahroni JH, et al. The independent contributions of diabetic neuropathy and vasculopathy in foot ulceration. Diabetes Care 1995;18:216-219.

4. Pecoraro RE, Reiber GE, Burgess EM. Causal pathways to amputation: Basis for prevention. Diabetes Care 1990;13:513-521.

5. Bell-Krotoski JA, Tomancik E. The repeatability of testing with Semmes-Weinstein Monofilaments. J Hand Surg 1987;12A:155-161.

6. Armstrong DG, Lavery LA, Vela SA, Quebedeaux TL, Fleischli JG. Choosing a practical screening instrument to identify patients at risk for diabetic foot ulceration. Arch Int Med 1998;158:289-292. 
7. Mayfield JA, Sugarman JR. The use of the Semmes-Weinstein Monofilament and other threshold tests for preventing foot ulceration and amputation in persons with diabetes. J Fam Pract 2000;49(Suppl):s17s29.

8. Valk GD, de Sonnaville JJ, van Houtum WH, et al. The assessment of diabetic polyneuropathy in daily clinical practice: Reproducibility and validity of Semmes Weinstein Monofilaments examination and clinical neurological examination. Muscle Nerve 1997;20:116-118.

9. Bell-Krotoski JA, Buford WL. The force/time relationship of clinically used sensory testing instruments. J Hand Ther 1997;10,4:297-309.

10. Booth J, Young MJ. Differences in the performance of commercially available 10-g monofilaments. Diabetes Care 2000;23:984-988.

11. Hodge MC, Nathan D, Bach TM. Plantar pressure pain thresholds and touch sensitivity in rheumatoid arthritis. Foot Ankle Int 2009;30:1-9.

12. Modawal A, Fley J, Shukla R, Rudawsky D, Welge J, Yang J. Use of monofilament in the detection of foot lesions in older adults. J Foot Ankle Surg 2006;45:76-81.
13. Dyck PJ, O'Brien PC, Kosanke JL, Gillen DA, Karnes JL. A 4, 2, and 1 stepping algorithm for quick and accurate estimation of cutaneous sensation threshold. Neurology 1993;43:1508-1512.

14. Berquin $A D$, Lijesevic $V$, Blond $S$, Plaghki L. An adaptive procedure for routine measurement of light-touch sensitivity threshold. Muscle Nerve 2010;42:328-338.

15. Padberg FT Jr, Maniker AH, Carmel G, Pappas PJ, Silva MB Jr, Hobson RW 2nd. Sensory impairment: a feature of chronic venous insufficiency. J Vasc Surg 1999;30:836-42.

16. Gescheider GA, Thorpe JM, Goodarz J, Bolanowski SJ. The effects of skin temperature on the detection and discrimination of tactile stimulation. Somatosens Mot Res 1997;14:181-188.

17. Jemec GB, Jemec B, Jemec BI, Serup J. The effect of superficial hydration on the mechanical properties of human skin in vivo: Implications for plastic surgery. Plast Reconstr Surg 1990;85:100-103.

18. Brand PW, Hollister AM. Clinical Mechanics of the Hand. 3rd ed. St Louis, MO: Mosby; 1999, 231-232. 